ORIGINAL ARTICLE

\title{
Trends of accuracy of clinical diagnoses of the basic cause of death in a university hospital
}

\author{
M H C Grade, S Zucoloto, J K Kajiwara, M T P Fernandes, L G F Couto, S B Garcia
}

J Clin Pathol 2004;57:369-373. doi: 10.1136/jcp.2003.013235

See end of article for authors' affiliations ....................

Correspondence to: Dr M H C Grade, Department of Pathology, Ribeirão Preto Medical School, University of São Paulo, Av. Bandeirantes, 3900 - Campus FMRP, USP, 14.049-000, Ribeirão Preto, Brazil; marcio@rpa.fmrp.usp.br

Accepted for publication 18 November 2003

\begin{abstract}
Aims: To determine the agreement between clinical and necropsy diagnoses of the basic cause of death, and to compare the results with those obtained in a previous study carried out at the same university hospital.

Methods: In total, 4828 necropsies, performed between 1990 and 1995 in the University Hospital of the Faculty of Medicine of Ribeirão Preto, University of São Paulo, Brazil, were reviewed. Examinations were concluded at the macroscopic part of the necropsy in nearly $35 \%$ of the cases. Statistical analysis was carried out using the $\kappa$ coefficient comparing the clinical diagnosis and the diagnosis obtained after necropsy. The jackknife method was used to identify comparable $\kappa$ values for the comparison of the two periods.

Results: Compared with the 1978-80 period, a significant increase in diagnostic agreement was seen for the group submitted to complete necropsy, whereas no similar increase was detected when only the macroscopic step was analysed.

Conclusions: There was a discrete tendency to an improved correlation between clinical and postmortem data stated by full necropsy analysis. The findings show that microscopic analysis remains important to confirm the cause of death in many cases. Diagnostic discrepancies remained high, and therefore complete necropsy continues to be an essential instrument for the assessment of clinical diagnosis.
\end{abstract}

S nce the publication of a study that related deaths to alterations in normal anatomy, ${ }^{1}$ necropsy has been established as an effective method to identify new nosological entities and to improve the investigation of known pathological diseases. ${ }^{2}{ }^{3}$ In addition to diagnostic and therapeutic errors, various diseases and their mechanisms have been established since then, and therapeutic methods have been developed based on postmortem data. ${ }^{4}$

Although necropsies are thought to be of crucial importance for medical teaching and for insuring the quality of medical care, ${ }^{5}$ a decline in the number of necropsies has been reported in several hospitals. ${ }^{6-10}$

The diagnostic process is a complex interaction between knowledge, abilities, and technical procedures under conditions of uncertainty. ${ }^{11}$ The technology available for diagnostic procedures has made great advances during the past few decades, particularly in terms of resources for diagnostic imaging techniques, such as computed tomography, ultrasonography, and nuclear magnetic resonance. However, these new technologies have also contributed to false positive and false negative diagnoses. ${ }^{12}$ Considering necropsy as the gold standard for diagnostic confirmation, studies carried out during the past 20 years have shown important disagreement and have failed to show a significant increase in diagnostic agreement in the assessment of pathologies, groups of pathologies, groups of patients (neonatal, paediatric, elderly, and psychiatric patients), or even different locations, such as intensive care units and hospitals affiliated or not with a university. ${ }^{13-23}$

"The technology available for diagnostic procedures has made great advances during the past few decades, particularly in terms of resources for diagnostic imaging techniques"

Another relevant aspect concerns the mortality statistics obtained from death certificate data. The accuracy of this information should be assessed and some studies have already demonstrated severe inaccuracies in death certificates. ${ }^{24-26}$

The aim of our present study was to determine the diagnostic agreement between clinical and necropsy diagnoses, and the role of macroscopic analysis in postmortem examination. On the basis of the results obtained, we also intended to assess the trends of accuracy of the clinical diagnoses made at our institution by comparing the results with those obtained in a similar study carried out during the period from 1978 to $1980 .^{27}$

\section{MATERIALS AND METHODS}

Providing medical care at the primary, secondary, tertiary, and emergency level, the University Hospital of the Faculty of Medicine of Ribeirão Preto, University of São Paulo (HCFMRP-USP), Brazil, is a public teaching hospital and considered to be a reference centre, where a high proportion of those patients who die are submitted to necropsy.

Necropsies carried out at HCFMRP-USP during the period from 1990 to 1995 were analysed. Our study was approved by the research ethics committee of the institution. Necropsies were performed by trainee pathologists, under direct supervision of the teaching staff of the department of pathology. Necropsies are not carried out until the medical chart of the deceased and the necropsy request are available. This request contains a clinical/laboratory resume and the International Model of Death Certificate, which has been filled out by the clinician.

The basic cause of death was identified within the sequence of morbid events based on three sources: necropsy

Abbreviations: HCFMRP-USP, University Hospital of the Faculty of Medicine of Ribeirão Preto, University of São Paulo; ICD, International Classification of Diseases 
request (clinical diagnosis), death certificate filled out after macroscopic examination (macroscopic diagnosis), and the necropsy report, including histological, biochemical, and serological tests, which were necessary for the identification of the main disease responsible for the process that resulted in death (final diagnosis).

Each diagnosis was coded according to the categories of three characters of the 10th manual of the International Classification of Diseases (ICD-10).$^{28}$ The data obtained were grouped according to the 21 categories of diseases of the ICD (table 1). Still births, fetal deaths, and deaths of children aged less than 1 year were excluded from the analysis.

The population analysed was divided into three groups: group 1, comparison of the basic cause of death based on the necropsy request and that obtained after macroscopic examination; group 2, comparison of the clinical diagnosis and the final one, which includes complementary investigations, such as microscopy; and group 3, comparison of the macroscopic step with the final diagnosis.

The $\kappa$ coefficient of agreement was calculated to compare the degree of diagnostic agreement between the groups. We regrouped the diagnoses according to the ninth revision of the ICD. ${ }^{29}$ The data were then compared statistically with those from 1978-80, ${ }^{27}$ using the jackknife method for the comparison of two independent $\kappa$ values, except for group 3 , because this comparison was not performed in the previous study.

\section{RESULTS}

In total, 8443 deaths occurred at HCFMRP-USP between 1990 and 1995, with $77.75 \%$ of those who died being submitted to necropsy. Among them, in addition to the categories excluded from our study as mentioned earlier, there were several cases for which public organisations other than the HCFMRP-USP were responsible as a result of legal requirements, such as the Legal Medicine Institute, even though the death had occurred at our hospital.

For the remaining 4828 necropsies, the three diagnoses were not available for all cases. Examinations were concluded

Table 1 Disease groups of the manual of the 10th revision of the International Classification of Diseases (ICD) distributed according to the 21 categories

ICD

category Disease group

1 Some infectious and parasitic diseases

Neoplasms

Blood disorders, disorders of the haemopoietic organs, some immune disorders

Endocrine, nutritional, and metabolic diseases

Mental and behavioural disorders

Nervous system diseases

Diseases of the eyes and adnexa

Diseases of the ear and mastoid apophysis

Diseases of the circulatory system

Diseases of the respiratory tract

Diseases of the digestive tract

Diseases of the skin and of the subcutaneous layer

Diseases of the osteomuscular system and connective tissue

Diseases of the genitourinary tract

Pregnancy, delivery, and puerperium

Some disorders originating during the perinatal period

Congenital malformations, deformities, and chromosome aberrations

Symptoms, signs, and abnormal findings upon clinical and laboratory examination not classified elsewhere

Lesions, poisoning, and some consequences of external causes

External causes of morbidity and mortality

Factors influencing the health status and contact with health services at the macroscopic part of the necropsy in almost 35\% of the cases. Another factor for the lack of diagnostic data was failure to fill out the documents or inability to read the diagnosis because it was written in an illegible manner.

For group 1 (4491 cases), the $\kappa$ coefficient of agreement was 0.679 , with a variance of $3.62 \times 10^{-5}$; for group $2(2901$ cases), $\kappa$ was 0.645 , with a variance of $5.67 \times 10^{-5}$; and for group 3 (3096 cases), $\kappa$ was 0.915, with a variance of $5.69 \times 10^{-5}$.

The results obtained for each group are presented according to the distribution of the diagnoses within the different ICD-10 categories, and are shown in tables 2-4.

Regrouping of the present data according to the disease categories of the ninth revision of the ICD (table 5) permitted statistical comparison between these independent variables using the jackknife method to identify comparable $\kappa$ values for the comparison of the two periods (table 6). Table 7 shows a comparison of the sensitivity and specificity for group 2 .

\section{DISCUSSION}

In our present study, diseases of the circulatory system were found to be the main cause of death (1573), followed by neoplasms (790), and infectious and parasitic diseases (414), as stated in the death certificate. This finding characterises HCFMRP-USP as a reference centre in the region with a large number of deaths caused by cancer; however, it should be emphasised that no data were computed regarding the many deaths caused by AIDS, which would belong in the category of infectious and parasitic diseases as basic cause of death, because the pathology service of the hospital did not carry out a necropsy on most of these patients during our study period.

Analysis of the levels of agreement between the groups showed a lower $\kappa$ value for group 2 than for group 1, which might be explained by the easier detection of discordant findings when a more rigorous method of analysis is used.

The level of agreement between macroscopic diagnosis and the final diagnosis after microscopic examination was highly significant (0.915). The pathology service of HCFMRP-USP frequently uses this type of examination, which is only disregarded when the macroscopic findings make the diagnosis obvious. This is because we are a teaching hospital, and microscopic examination is carried out routinely in many cases. Nevertheless, despite an apparent high rate of agreement between macroscopic and microscopic findings, only microscopic examination revealed the tendency towards improvement in the level of diagnostic agreement seen in our study.

Zaitoun and Fernandez ${ }^{30}$ also showed that histological examination significantly contributed to the final diagnosis in $5 \%$ of their series, whereas other minor pathological findings contributed in $6 \%$ of their cases.

By regrouping the diseases according to the ninth revision of the ICD, we also compared, as suggested by Saracci, ${ }^{31}$ the diagnostic sensitivity of complete necropsy. The results showed that most comparable groups maintained almost the same level of diagnostic sensitivity. The following sensitivity rates were observed for the categories with the largest numbers of deaths: diseases of the circulatory system ( $74.1 \%$ in the previous study $v 75.3 \%$ in our present study), neoplasms $(81.6 \% \vee 81.6 \%)$, and infectious and parasitic diseases $(59.4 \% \vee 61.4 \%)$. It is worth mentioning that the sensitivity for the last category might increase if necropsies of patients with a diagnosis of AIDS were carried out and computed-this diagnosis as a basic cause of death shows a higher chance of agreement because our hospital is a reference centre in the region for the follow up of these patients. 
Table 2 Distribution of patients who died at HCFMRP-USP from 1990 to 1995 according to the 21 diagnostic categories of the ICD-10 based on the basic cause of death on the CD and that obtained after MD

\begin{tabular}{|c|c|c|c|c|c|c|c|c|c|c|c|c|c|c|c|c|c|c|c|c|c|c|}
\hline \multirow[b]{2}{*}{$C D$} & \multicolumn{22}{|l|}{ MD } \\
\hline & 1 & 2 & 3 & 4 & 5 & 6 & 7 & 8 & 9 & 10 & 11 & 12 & 13 & 14 & 15 & 16 & 17 & 18 & 19 & 20 & 21 & Total \\
\hline 1 & 272 & 7 & 0 & 7 & 2 & 13 & 0 & 0 & 40 & 14 & 21 & 2 & 0 & 4 & 1 & 0 & 0 & 1 & 4 & 0 & 0 & 388 \\
\hline 2 & 7 & 669 & 0 & 0 & 4 & 1 & 0 & 0 & 10 & 3 & 16 & 0 & 0 & 3 & 0 & 0 & 0 & 0 & 0 & 0 & 0 & 713 \\
\hline 3 & 1 & 3 & 50 & 0 & 0 & 1 & 0 & 0 & 8 & 1 & 6 & 0 & 1 & 1 & 1 & 0 & 0 & 0 & 0 & 0 & 0 & 73 \\
\hline 4 & 4 & 2 & 1 & 97 & 10 & 1 & 0 & 0 & 32 & 1 & 9 & 0 & 0 & 2 & 0 & 0 & 0 & 0 & 1 & 0 & 0 & 160 \\
\hline 5 & 3 & 5 & 0 & 1 & 200 & 1 & 0 & 0 & 12 & 3 & 10 & 0 & 0 & 2 & 0 & 0 & 0 & 0 & 0 & 0 & 0 & 237 \\
\hline 6 & 7 & 5 & 1 & 2 & 4 & 105 & 0 & 4 & 13 & 7 & 1 & 1 & 0 & 1 & 0 & 0 & 0 & 0 & 0 & 0 & 0 & 151 \\
\hline 7 & 0 & 0 & 0 & 0 & 0 & 0 & 0 & 0 & 0 & 0 & 0 & 0 & 0 & 0 & 0 & 0 & 0 & 0 & 0 & 0 & 0 & 0 \\
\hline 8 & 0 & 0 & 0 & 0 & 0 & 0 & 0 & 3 & 0 & 0 & 0 & 0 & 0 & 0 & 0 & 0 & 0 & 0 & 0 & 0 & 0 & 3 \\
\hline 9 & 60 & 23 & 4 & 9 & 16 & 18 & 0 & 0 & 1206 & 32 & 31 & 2 & 1 & 13 & 1 & 0 & 5 & 4 & 4 & 0 & 0 & 1429 \\
\hline 10 & 38 & 38 & 3 & 12 & 22 & 9 & 0 & 0 & 174 & 223 & 29 & 1 & 10 & 16 & 1 & 0 & 1 & 8 & 6 & 0 & 0 & 591 \\
\hline 11 & 14 & 23 & 0 & 3 & 57 & 2 & 0 & 0 & 25 & 7 & 249 & 0 & 0 & 9 & 0 & 0 & 0 & 0 & 0 & 0 & 0 & 389 \\
\hline 12 & 1 & 0 & 0 & 0 & 0 & 0 & 0 & 0 & 1 & 1 & 0 & 11 & 1 & 0 & 0 & 0 & 0 & 0 & 0 & 0 & 0 & 15 \\
\hline 13 & 0 & 1 & 1 & 0 & 0 & 0 & 0 & 0 & 2 & 1 & 0 & 0 & 23 & 0 & 0 & 0 & 0 & 0 & 0 & 0 & 0 & 28 \\
\hline 14 & 3 & 8 & 2 & 8 & 2 & 1 & 0 & 0 & 23 & 6 & 3 & 0 & 2 & 89 & 1 & 0 & 1 & 1 & 1 & 0 & 0 & 151 \\
\hline 15 & 0 & 0 & 0 & 0 & 0 & 0 & 0 & 0 & 0 & 0 & 0 & 0 & 0 & 0 & 21 & 0 & 0 & 0 & 0 & 0 & 0 & 21 \\
\hline 16 & 0 & 0 & 0 & 0 & 0 & 0 & 0 & 0 & 0 & 0 & 0 & 0 & 0 & 0 & 0 & 1 & 0 & 0 & 0 & 0 & 0 & 1 \\
\hline 17 & 0 & 0 & 0 & 0 & 0 & 0 & 0 & 0 & 1 & 0 & 0 & 0 & 0 & 0 & 0 & 0 & 23 & 0 & 0 & 0 & 0 & 24 \\
\hline 18 & 2 & 4 & 0 & 1 & 4 & 0 & 0 & 0 & 8 & 1 & 10 & 0 & 0 & 1 & 0 & 0 & 0 & 11 & 1 & 0 & 0 & 43 \\
\hline 19 & 2 & 2 & 0 & 0 & 2 & 1 & 0 & 0 & 18 & 1 & 3 & 0 & 0 & 0 & 0 & 0 & 0 & 3 & 41 & 0 & 0 & 73 \\
\hline 20 & 0 & 0 & 0 & 0 & 0 & 0 & 0 & 0 & 0 & 0 & 0 & 0 & 0 & 0 & 0 & 0 & 0 & 0 & 0 & 1 & 0 & 1 \\
\hline 21 & 0 & 0 & 0 & 0 & 0 & 0 & 0 & 0 & 0 & 0 & 0 & 0 & 0 & 0 & 0 & 0 & 0 & 0 & 0 & 0 & 0 & 0 \\
\hline Total & 414 & 790 & 62 & 140 & 323 & 153 & 0 & 7 & 1573 & 301 & 388 & 17 & 38 & 141 & 26 & 1 & 30 & 28 & 58 & 1 & 0 & 4491 \\
\hline
\end{tabular}

Some categories of the ICD showed a lower diagnostic sensitivity than others, especially category 18 (symptoms, signs, and abnormal findings upon clinical and laboratory examination not classified elsewhere). On the basis of the description of this category, it is assumed that an exact diagnosis was not made, but that only alterations produced by the disease were considered, represented by clear examinations, signs, and symptoms.

With respect to improvement in diagnostic sensitivity, a significant increase from $38.6 \%$ to $61.3 \%$ was observed for the category of mental and behavioral disorders, a finding mainly demonstrating the concern of clinicians to include chronic alcoholism as a basic cause of death. In the category of respiratory diseases, diagnostic sensitivity increased significantly from $35.7 \%$ to $76.6 \%$. This is because of the greater care in excluding non-specific pneumonia as a basic cause of death, in addition to the significant improvement in diagnostic procedures during the past few years. A significant increase in sensitivity from $37 \%$ to $60 \%$ was also seen for the category of congenital malformations, deformities, and chromosome aberrations, indicating improvement in the assessment of these cases, in addition to the diagnostic procedures used in this area.

A decrease in diagnostic sensitivity was seen for the category of diseases of the osteomuscular system and connective tissue $(81.8 \%$ to $50.0 \%)$. This finding probably

Table 3 Distribution of patients who died at HCFMRP-USP from 1990 to 1995 according to the 21 diagnostic categories of the ICD-10 based on diagnosis of the basic cause of death on the CD and the FD

\begin{tabular}{|c|c|c|c|c|c|c|c|c|c|c|c|c|c|c|c|c|c|c|c|c|c|c|}
\hline \multirow[b]{2}{*}{$C D$} & \multicolumn{22}{|l|}{ FD } \\
\hline & 1 & 2 & 3 & 4 & 5 & 6 & 7 & 8 & 9 & 10 & 11 & 12 & 13 & 14 & 15 & 16 & 17 & 18 & 19 & 20 & 21 & Total \\
\hline 1 & 172 & 9 & 0 & 5 & 2 & 1 & 0 & 0 & 31 & 5 & 19 & 1 & 1 & 2 & 0 & 0 & 0 & 1 & 5 & 0 & 0 & 254 \\
\hline 2 & 4 & 390 & 0 & 2 & 3 & 1 & 0 & 0 & 9 & 1 & 8 & 0 & 0 & 2 & 0 & 0 & 0 & 1 & 0 & 0 & 0 & 421 \\
\hline 3 & 0 & 5 & 21 & 0 & 0 & 1 & 0 & 0 & 3 & 1 & 4 & 0 & 2 & 0 & 1 & 0 & 0 & 0 & 0 & 0 & 0 & 38 \\
\hline 4 & 4 & 0 & 1 & 48 & 9 & 5 & 0 & 0 & 19 & 2 & 3 & 0 & 0 & 1 & 0 & 0 & 0 & 0 & 1 & 0 & 0 & 93 \\
\hline 5 & 6 & 2 & 0 & 0 & 147 & 0 & 0 & 0 & 7 & 2 & 5 & 0 & 0 & 2 & 0 & 0 & 0 & 0 & 0 & 0 & 0 & 171 \\
\hline 6 & 6 & 3 & 0 & 2 & 3 & 62 & 0 & 2 & 9 & 3 & 1 & 1 & 0 & 1 & 0 & 1 & 0 & 0 & 0 & 0 & 0 & 94 \\
\hline 7 & 0 & 0 & 0 & 0 & 0 & 0 & 0 & 0 & 0 & 0 & 0 & 0 & 0 & 0 & 0 & 0 & 0 & 0 & 0 & 0 & 0 & 0 \\
\hline 8 & 0 & 0 & 0 & 0 & 0 & 0 & 0 & 3 & 0 & 0 & 0 & 0 & 0 & 0 & 0 & 0 & 0 & 0 & 0 & 0 & 0 & 3 \\
\hline 9 & 36 & 15 & 3 & 7 & 9 & 10 & 0 & 0 & 807 & 20 & 21 & 1 & 1 & 10 & 1 & 0 & 4 & 3 & 4 & 0 & 0 & 952 \\
\hline 10 & 22 & 29 & 1 & 7 & 20 & 3 & 0 & 0 & 130 & 128 & 19 & 1 & 7 & 9 & 1 & 0 & 1 & 5 & 9 & 0 & 0 & 392 \\
\hline 11 & 22 & 18 & 0 & 1 & 43 & 1 & 0 & 0 & 19 & 2 & 148 & 0 & 0 & 10 & 0 & 0 & 0 & 0 & 0 & 0 & 0 & 264 \\
\hline 12 & 1 & 0 & 0 & 1 & 0 & 0 & 0 & 0 & 1 & 0 & 0 & 9 & 1 & 0 & 0 & 0 & 0 & 0 & 0 & 0 & 0 & 13 \\
\hline 13 & 1 & 0 & 0 & 0 & 0 & 1 & 0 & 0 & 2 & 0 & 0 & 0 & 13 & 0 & 0 & 0 & 0 & 0 & 0 & 0 & 0 & 17 \\
\hline 14 & 3 & 3 & 2 & 6 & 0 & 0 & 0 & 0 & 17 & 3 & 4 & 0 & 1 & 53 & 2 & 0 & 1 & 0 & 1 & 0 & 0 & 96 \\
\hline 15 & 1 & 0 & 0 & 0 & 0 & 0 & 0 & 0 & 1 & 0 & 0 & 0 & 0 & 0 & 13 & 0 & 0 & 0 & 0 & 0 & 0 & 15 \\
\hline 16 & 0 & 0 & 0 & 0 & 0 & 0 & 0 & 0 & 0 & 0 & 0 & 0 & 0 & 0 & 0 & 0 & 0 & 0 & 0 & 0 & 0 & 0 \\
\hline 17 & 0 & 0 & 0 & 0 & 0 & 0 & 0 & 0 & 0 & 0 & 1 & 0 & 0 & 0 & 0 & 0 & 9 & 0 & 0 & 0 & 0 & 10 \\
\hline 18 & 1 & 3 & 1 & 1 & 1 & 0 & 0 & 0 & 7 & 0 & 4 & 0 & 0 & 0 & 0 & 0 & 0 & 6 & 1 & 0 & 0 & 25 \\
\hline 19 & 1 & 1 & 0 & 0 & 3 & 0 & 0 & 0 & 10 & 0 & 2 & 0 & 0 & 0 & 0 & 0 & 0 & 1 & 24 & 0 & 0 & 42 \\
\hline 20 & 0 & 0 & 0 & 0 & 0 & 0 & 0 & 0 & 0 & 0 & 0 & 0 & 0 & 0 & 0 & 0 & 0 & 0 & 0 & 1 & 0 & 1 \\
\hline 21 & 0 & 0 & 0 & 0 & 0 & 0 & 0 & 0 & 0 & 0 & 0 & 0 & 0 & 0 & 0 & 0 & 0 & 0 & 0 & 0 & 0 & 0 \\
\hline Total & 280 & 478 & 29 & 80 & 240 & 85 & 0 & 5 & 1072 & 167 & 239 & 13 & 26 & 90 & 18 & 1 & 15 & 17 & 45 & 1 & 0 & 2901 \\
\hline
\end{tabular}

Cases for which no microscopic investigation was carried out were excluded.

CD, necropsy request; FD, final necropsy report; HCFMRP-USP, University Hospital of the Faculty of Medicine of Ribeirão Preto, University of São Paulo; ICD, International Classification of Diseases. 
Table 4 Distribution of patients who died at HCFMRP-USP during the period from 1990 to 1995 according to the 21 diagnostic categories of the ICD-10 based on the necropsy diagnosis of the basic cause of death obtained after MD and FD

\begin{tabular}{|c|c|c|c|c|c|c|c|c|c|c|c|c|c|c|c|c|c|c|c|c|c|c|}
\hline & FD & & & & & & & & & & & & & & & & & & & & & \\
\hline$M D$ & 1 & 2 & 3 & 4 & 5 & 6 & 7 & 8 & 9 & 10 & 11 & 12 & 13 & 14 & 15 & 16 & 17 & 18 & 19 & 20 & 21 & Total \\
\hline 1 & 255 & 3 & 0 & 2 & 2 & 1 & 0 & 0 & 3 & 0 & 4 & 0 & 0 & 0 & 0 & 0 & 0 & 0 & 1 & 0 & 0 & 271 \\
\hline 2 & 1 & 486 & 0 & 1 & 1 & 1 & 0 & 0 & 4 & 1 & 0 & 0 & 0 & 0 & 0 & 0 & 0 & 1 & 0 & 0 & 0 & 496 \\
\hline 3 & 0 & 1 & 29 & 0 & 0 & 1 & 0 & 0 & 1 & 0 & 0 & 0 & 0 & 0 & 0 & 0 & 0 & 0 & 0 & 0 & 0 & 32 \\
\hline 4 & 1 & 0 & 0 & 75 & 5 & 5 & 0 & 0 & 5 & 1 & 0 & 0 & 0 & 0 & 0 & 0 & 0 & 1 & 1 & 0 & 0 & 94 \\
\hline 5 & 7 & 1 & 0 & 0 & 235 & 1 & 0 & 0 & 4 & 1 & 3 & 0 & 0 & 0 & 0 & 0 & 0 & 0 & 0 & 0 & 0 & 252 \\
\hline 6 & 4 & 3 & 0 & 0 & 0 & 75 & 0 & 0 & 8 & 2 & 0 & 0 & 0 & 0 & 0 & 1 & 0 & 1 & 0 & 0 & 0 & 94 \\
\hline 7 & 0 & 0 & 0 & 0 & 0 & 0 & 0 & 0 & 0 & 0 & 0 & 0 & 0 & 0 & 0 & 0 & 0 & 0 & 0 & 0 & 0 & 0 \\
\hline 8 & 0 & 0 & 0 & 0 & 0 & 0 & 0 & 6 & 0 & 0 & 0 & 0 & 0 & 0 & 0 & 0 & 0 & 0 & 0 & 0 & 0 & 6 \\
\hline 9 & 8 & 4 & 0 & 1 & 2 & 1 & 0 & 0 & 1105 & 8 & 2 & 0 & 1 & 4 & 0 & 0 & 1 & 0 & 2 & 0 & 0 & 1139 \\
\hline 10 & 6 & 7 & 0 & 1 & 3 & 0 & 0 & 0 & 9 & 159 & 1 & 0 & 1 & 0 & 0 & 0 & 0 & 0 & 4 & 0 & 0 & 191 \\
\hline 11 & 10 & 7 & 0 & 2 & 13 & 0 & 0 & 0 & 1 & 0 & 239 & 0 & 0 & 2 & 1 & 0 & 0 & 1 & 0 & 0 & 0 & 276 \\
\hline 12 & 0 & 0 & 0 & 1 & 0 & 1 & 0 & 0 & 0 & 0 & 0 & 13 & 0 & 0 & 0 & 0 & 0 & 0 & 0 & 0 & 0 & 15 \\
\hline 13 & 0 & 0 & 0 & 0 & 0 & 1 & 0 & 0 & 0 & 1 & 0 & 0 & 25 & 0 & 0 & 0 & 0 & 0 & 0 & 0 & 0 & 27 \\
\hline 14 & 0 & 0 & 1 & 1 & 1 & 0 & 0 & 0 & 2 & 1 & 2 & 0 & 0 & 91 & 1 & 0 & 0 & 0 & 1 & 0 & 0 & 101 \\
\hline 15 & 1 & 0 & 0 & 0 & 0 & 0 & 0 & 0 & 1 & 0 & 0 & 0 & 0 & 0 & 20 & 0 & 0 & 0 & 0 & 0 & 0 & 22 \\
\hline 16 & 0 & 0 & 0 & 0 & 0 & 0 & 0 & 0 & 0 & 0 & 0 & 0 & 0 & 0 & 0 & 0 & 0 & 0 & 0 & 0 & 0 & 0 \\
\hline 17 & 0 & 0 & 0 & 0 & 0 & 0 & 0 & 0 & 0 & 0 & 1 & 0 & 0 & 0 & 0 & 0 & 14 & 0 & 0 & 0 & 0 & 15 \\
\hline 18 & 1 & 1 & 0 & 0 & 0 & 0 & 0 & 0 & 3 & 1 & 0 & 0 & 0 & 0 & 0 & 0 & 0 & 16 & 0 & 0 & 0 & 22 \\
\hline 19 & 0 & 0 & 0 & 0 & 1 & 0 & 0 & 0 & 1 & 0 & 0 & 0 & 0 & 0 & 0 & 0 & 0 & 0 & 40 & 0 & 0 & 42 \\
\hline 20 & 0 & 0 & 0 & 0 & 0 & 0 & 0 & 0 & 0 & 0 & 0 & 0 & 0 & 0 & 0 & 0 & 0 & 0 & 0 & 1 & 0 & 1 \\
\hline 21 & 0 & 0 & 0 & 0 & 0 & 0 & 0 & 0 & 0 & 0 & 0 & 0 & 0 & 0 & 0 & 0 & 0 & 0 & 0 & 0 & 0 & 0 \\
\hline Total & 294 & 513 & 30 & 84 & 263 & 87 & 0 & 6 & 1147 & 175 & 252 & 13 & 27 & 97 & 22 & 1 & 15 & 20 & 49 & 1 & 0 & 3096 \\
\hline
\end{tabular}

FD, microscopic examination; HCFMRP-USP, University Hospital of the Faculty of Medicine of Ribeirão Preto, University of São Paulo; ICD, International

Classification of Diseases; MD, macroscopic examination.

reflects the small number of deaths in this group (five each year) and is associated with inadequate reporting of the basic causes of death, because in various cases respiratory problems were classified as the direct cause of death. A decrease $(71.4 \%$ to $58.9 \%)$ in diagnostic sensitivity, although not highly significant, was also seen for diseases of the genitourinary tract.

Improvement in the level of agreement with the final diagnosis was seen compared with that found in a study carried out in our hospital at the end of the $1970,{ }^{27}$ which was not the case when the diagnoses on the death certificates were compared.

This tendency towards improvement in the level of diagnostic agreement has rarely been reported in the literature, as shown by Sonderegger-Iseli et al and Tsujimura and colleagues ${ }^{11}{ }^{32}$ in a review of similar studies

Table 5 The 17 categories of diseases of the ninth revision of the International Classification of Diseases (ICD), 1975

\begin{tabular}{ll}
\hline ICD & \\
category & Group of diseases \\
\hline 1 & Infectious and parasitic diseases \\
2 & Neoplasms \\
3 & Endocrine, nutritional, and metabolic diseases and immunity \\
& disorders \\
4 & Diseases of the blood and bloodforming organs \\
5 & Mental disorders \\
6 & Diseases of the nervous system and sense organs \\
7 & Diseases of the circulatory system \\
8 & Diseases of the respiratory system \\
9 & Diseases of the digestive system \\
10 & Diseases of the genitourinary system \\
11 & Complications of pregnancy, childbirth, and the puerperium \\
12 & Diseases of the skin and subcutaneous tissue \\
13 & Diseases of the musculoskeletal system and connective tissue \\
14 & Congenital anomalies \\
15 & Certain conditions originating in the perinatal period \\
16 & Symptoms, signs, and ill defined conditions \\
17 & Injury and poisoning \\
\hline
\end{tabular}

in the literature. Recently, in Brazil Coradazzi et al presented a study comparing two periods (1975-82 v 1992-6), and showed an improvement in diagnostic agreement of $72.9 \%$ to $79.4 \%$ for the basic cause of death. ${ }^{33}$

This trend was seen in our pathology service as a result of an improvement in the assessment of the basic cause of death by clinicians, which was especially evident with respect to chronic alcoholism as the basic cause. It should be mentioned, however, that advances in diagnostic techniques might also influence this tendency. However, this improvement seems to be small in view of the many advances that occurred during the 1980s and at the beginning of the 1990s. Therefore, it is important to emphasise that disagreements continue to occur, as shown in several studies. ${ }^{13-23}$

In this respect, each procedure that helps in the analysis of diagnostic discrepancies should be emphasised. Our findings show that microscopic analysis remains important to establish or confirm the cause of death in many cases. Further studies are necessary to discern those situations in which microscopic analysis is most useful.

Quality control is a highly important objective in health care and one of the main reasons why necropsy continues to be essential for improving medical care. ${ }^{34}$ Necropsy continues to be the gold standard for the determination of the cause of death and associated diseases, and is necessary for the assessment of quality of care in health services. It should be

Table 6 Values of $\kappa$ ( $\kappa$ for comparison) for study groups 1 and 2 for the periods $1978-80$ and $1990-5$

\begin{tabular}{lll}
\hline & Group 1 & Group 2* \\
\hline ki 1978-80 & 0.661 & 0,601 \\
Variance & $2.75 \times 10^{-4}$ & $3 \times 10^{-4}$ \\
кi 1990-5 & 0.661 & 0.646 \\
Variance & $5.85 \times 10^{-5}$ & $1 \times 10^{-4}$ \\
\hline
\end{tabular}

*Significant difference for values in a Z table score.

Group 1, comparison of the basic cause of death based on the necropsy request and that obtained after macroscopic examination; group 2,

comparison of the clinical diagnosis and the final one, which includes complementary investigations, such as microscopy. 
Table 7 Sensitivity and specificity comparison for the 17 categories of ICD-9, 1978-80 v 1990-5, group 2

\begin{tabular}{|c|c|c|c|c|}
\hline \multirow{2}{*}{$\begin{array}{l}\text { ICD category of } \\
\text { disease }\end{array}$} & \multicolumn{2}{|l|}{$1978-80$} & \multicolumn{2}{|l|}{$1990-5$} \\
\hline & Sens $(\%)$ & Spec (\%) & Sens (\%) & Spec $(\%)$ \\
\hline 1 & 59.4 & 95.6 & 61.4 & 97.2 \\
\hline 2 & 81.6 & 97.9 & 81.6 & 98.9 \\
\hline 3 & 55.0 & 98.2 & 60.0 & 98.4 \\
\hline 4 & 83.3 & 99.2 & 72.4 & 99.4 \\
\hline 5 & 38.6 & 99.1 & 61.3 & 99.2 \\
\hline 6 & 75.0 & 98.6 & 74.4 & 99.1 \\
\hline 7 & 74.1 & 88.2 & 75.3 & 95.0 \\
\hline 8 & 35.7 & 96.1 & 76.6 & 90.9 \\
\hline 9 & 55.6 & 96.6 & 61.9 & 96.0 \\
\hline 10 & 71.4 & 96.8 & 58.9 & 98.5 \\
\hline 11 & 68.4 & 100.0 & 72.2 & 99.9 \\
\hline 12 & 50.0 & 99.8 & 69.2 & 99.9 \\
\hline 13 & 81.8 & 99.7 & 50.0 & 99.9 \\
\hline 14 & 37.0 & 99.7 & 60.0 & 100.0 \\
\hline 15 & 50.0 & 99.8 & 0.0 & 100.0 \\
\hline 16 & 25.0 & 97.3 & 35.3 & 99.3 \\
\hline 17 & 70.8 & 98.5 & 54.3 & 99.2 \\
\hline
\end{tabular}

Group 2, comparison of the clinical diagnosis and the final one, which includes complementary investigations, such as microscopy.

ICD-9, ninth revision of the International Classification of Diseases; sens, sensitivity; spec, specificity.

\section{Take home messages}

- When the periods $1978-80$ and $1990-5$ were compared there was an improved correlation between clinical and postmortem data in the group submitted to complete necropsy, although this was not the case when only the macroscopic step was analysed

- Microscopic analysis remains important to confirm the cause of death in many cases

- Diagnostic discrepancies remained high, so that complete necropsy continues to be essential for the assessment of clinical diagnosis

remembered that necropsy is not free of errors, but provides the best diagnosis of the cause of death.

\section{Authors' affiliations}

M H C Grade, S Zucoloto, J K Kajiwara, M T P Fernandes, L G F Couto, S B Garcia, Department of Pathology, Ribeirão Preto Medical School, University of São Paulo, Av. Bandeirantes, 3900 - Campus FMRP, USP, 14.049-000, Ribeirão Preto, Brazil

\section{REFERENCES}

1 King LS, Meehan MC. A history of autopsy. Am J Pathol 1973;73:514-44.

2 Silverberg SG. The autopsy and cancer. Arch Pathol Lab Med 1984; 108:476.

3 Wissler RW. The value of the autopsy for understanding cardiovascular disease. Arch Pathol Lab Med 1984;108:479.

4 Hill RB, Anderson RE. The autopsy-medical practice and public policy. Boston: Butterworth Publishers, 1988.

5 Goldman L, Sayson R, Robbins S, et al. The value of autopsy in three medical eras. N Engl J Med 1983;308:1000-5.

6 Cameron HM. The autopsy, past and present. J R Coll Physicians Lond 1984;18:236-9.

7 Goldman L. Diagnostic advances vs the value of autopsy. Arch Pathol Lab Med 1984;108:501-5.

8 Anderson NH, Shanks JH, McCluggage GW, et al. Necropsies in clinical audit. J Clin Pathol 1989:42:897-901.

9 Chute CG, Ballard DJ, Nemetz PN. Contribution of autopsy to populationbased cancer epidemiology: target intervention to improve ascertainment. IARC Sci Publ 1991;112:207-16.

10 Bõttiger LE. The post-mortem: its decline and fall [editorial]? J Intern Med 1992;231:99-101.

11 Sonderegger-lseli K, Burger S, Muntwyler J, et al. Diagnostic errors in three medical eras: a necropsy study. Lancet 2000;335:2027-31
12 McPhee SJ. The autopsy: an antidote to misdiagnosis. Medicine 1996;76:41-3.

13 Landefeld CS, Chren MM, Myers A, et al. Diagnostic yield of the autopsy in a university hospital and a community hospital. N Engl J Med 1988:318:1249-54.

14 Fernandez-Segoviano P, Lázaro A, Esteban A, et al. Autopsy as quality assurance in the intensive care unit. Crit Care Med 1988;16:683-5.

15 Blosser AS, Zimmerman HE, Stauffer JL. Do autopsies of critically ill patients reveal important findings that were clinically undetected? Crit Care Med 1998;26:1332-36.

16 Tai DYH, El-Bilbeisi H, Tewari S, et al. A study of consecutive autopsies in a medical ICU. Chest 2001;119:530-6.

17 Egerváry M, Szende B, Roe FJ, et al. Accuracy of clinical diagnosis of lung cancer in Budapest in an institute specializing in chest diseases. Pathol Res Pract 2000;196:761-6.

18 Tse GM, Lee JC. A 12-month review of autopsies performed at a universityaffiliated hospital in Hong-Kong. Hong Kong Med J 2000;6:190-4.

19 Juvin P, Teissiere F, Brion F, et al. Postoperative death and malpractice suits: is autopsy useful? Anesth Analg 2000;91:344-6.

20 Kumar P, Angst DB, Taxy J, et al. Neonatal autopsies: a 10-year experience. Arch Pediatr Adolesc Med 2000;154:38-42.

21 Kumar P, Angst DB, Taxy J, et al. Autopsies in children: are they still useful? Arch Pediatr Adolesc Med 1998; 152:558-63.

22 Salib E, Tadros G, Ambrose A. Autopsy in elderly psychiatric inpatients: a retrospective review of autopsy findings of deceased elderly psychiatric inpatients in North Cheshire 1980-1996. Med Sci Law 2000;40:20-7.

23 Twigg SJ, McCrirrick A, Sanderson PM. A comparison of post mortem findings with post hoc estimated clinical diagnoses of patients who died in a United Kingdom intensive care unit. Intensive Care Med 2001;27:706-10.

24 Cameron HM, McGoogan E. A prospective study of 1152 hospital autopsies: I. Inaccuracies in death certification. J Pathol 1981;133:273.

25 Kircher T, Nelson J, Burdo $\mathrm{H}$. The autopsy as a measure of accuracy of the death certificate. N Engl J Med 1985;313:1263-9.

26 Sington JD, Cottrell BJ. Analysis of the sensitivity of death certificates in 440 hospital deaths: a comparison with necropsy findings. J Clin Pathol 2002; 55:499-502.

27 Kajiwara JK, Zucoloto S, Manço ARX, et al. Accuracy of clinical diagnoses in a teaching hospital: a review of 997 autopsies. J Intern Med 1993;234:181-7.

28 International Classification of Diseases (ICD), 10th revision, 1989. Geneva: World Health Organisation, 1989.

29 International Classification of Diseases (ICD), 9th revision, 1975. Geneva: World Health Organisation, 1975.

30 Zaitoun AM, Fernandez C. The value of histological examination in the audit of hospital autopsies: a quantitative approach. Pathology 1998;30:100-4.

31 Saracci R. Is necropsy a valid monitor of clinical diagnosis performance? BMJ 1991;303:898-900.

32 Tsujimura T, Yamada $Y$, Kubo $M$, et al. Why couldn't an accurate diagnosis be made? An analysis of 1044 consecutive autopsy cases. Pathol Int 1999;49:408-10.

33 Coradazzi AL, Morganti ALC, Montenegro MRG. Discrepancies between clinical diagnoses and autopsy findings. Braz J Med Biol Res 2003;36:385-91.

34 Bayer-Garner IB, Fink LM, Lamps LW. Pathologists in a teaching institution assess the value of the autopsy. Arch Pathol Lab Med 2002;126:442-7. 